\title{
Variante Protrombótica C677T del Gen Metilenotetrahidrofolato Reductasa es un Biomarcador Molecular de Fallas de Implantación en Mujeres Chilenas Sometidas a Protocolos de Reproducción Asistida
}

\author{
Prothrombotic Variant C677T at the Methylenetetrahydrofolate Reductase Gene is a Molecular \\ Biomarker of Implantation Failure in Chilean Women Undergoing Assisted Reproductive Protocols
}

\author{
"Neftalí Guzmán; "María José Jara; "Leslie Morales; "Carol Sepúlveda; *Dina Guzmán; \\ **Hugo Henríquez; ${ }^{* * * *}$ Ricardo García; ${ }^{* * *}$ Juan Escalona \& ${ }^{* * *}$ Italo Ciuffardi
}

GUZMÁN, N.; JARA, M. J.; MORALES, L.; SEPÚLVEDA, C.; GUZMÁN, D.; HENRÍQUEZ, H.; GARCÍA, R.; ESCALONA, J. \& CIUFFARDI, I. Variante protrombótica C677T del gen metilenotetrahidrofolato reductasa es un biomarcador molecular de fallas de implantación en mujeres chilenas sometidas a protocolos de reproducción asistida. Int. J. Morphol., 28(1):65-69, 2010.

RESUMEN: Las fallas de implantación son consideradas una importante causa de infertilidad en mujeres sometidas a protocolos de Fecundación Asistida. Evidencia reciente sugiere que la presencia de variantes protrombóticas está asociada a diversos defectos obstétricos y falla reproductiva. Sin embargo, los resultados en diversas poblaciones son contradictorios. De acuerdo a esta evidencia, se evaluó la potencial asociación entre variantes protrombóticas y fallas de implantación en mujeres chilenas incluidas en protocolos de fecundación asistida. Un total de 180 mujeres, 80 pacientes sometidas a protocolos de reproducción asistida y 100 controles fueron incluidas en este estudio. La genotipificación molecular de variantes protrombóticas en genes candidatos fue realizada por PCR-RFLP. Observamos una paciente heterocigota para la variante F5 G1691A y ausencia total del polimorfismo F2 G20210A en pacientes y controles. La distribución genotípica y la distribución relativa de alelos del polimorfismo MTHFR C677T fueron significativamente diferentes entre pacientes y controles. Odds Ratio para fallas de implantación asociadas al genotipo homocigoto fue 2,78 (95\% IC 1,147 - 6,755; p 0,0199). En resumen, nuestros resultados sugieren que la variante MTHFR C677T constituye un biomarcador molecular de susceptibilidad a fallas de implantación población chilena.

PALABRAS CLAVE: Infertilidad; Fallas de implantación; Biomarcador.

\section{INTRODUCCIÓN}

La infertilidad es un problema de salud que afecta a un número creciente de mujeres en edad reproductiva (Zinaman et al., 1996), siendo la fecundación asistida una herramienta terapéutica comúnmente utilizada en el tratamiento de esta alteración. Sin embargo, las fallas de implantación constituyen uno de los principales inconvenientes en mujeres sometidas a procedimientos de fecundación asistida (Macklon et al., 2002; McIntyre et al., 2003).

Recientemente se ha descrito la asociación de variantes relacionadas a hipercoagulabilidad con alteraciones obstétricas y fallas de implantación posterior a procedimientos de reproducción asistida (Grandone et al., 2001; Villarreal et al., 2002; Buchholz \& Thaler, 2003; Wolf et al., 2003). Si bien los mecanismos responsables no están completamente dilucidados, la evidencia disponible permite sugerir que la presencia de variantes protrombóticas podría afectar la circulación útero-placentaria, desencadenando fallas reproductivas (Azem et al., 2004; Red-Horse et al., 2004).

En Chile, la evidencia científica disponible para la presencia de polimorfismos asociados a fallas reproductivas es escasa, por lo que el objetivo del estudio fue evaluar la potencial asociación de variantes protrombóticas con fallas de implantación en pacientes sometidas a procedimientos de fecundación asistida y controles.

\footnotetext{
* Laboratorio de Diagnóstico Molecular, Facultad de Ciencias de la Salud, Universidad San Sebastián, Chile.

*** Facultad de Medicina, Universidad Mayor, Santiago, Chile.

****Instituto de Medicina Reproductiva, Concepción, Chile.
} 
GUZMÁN, N.; JARA, M. J.; MORALES, L.; SEPÚLVEDA, C.; GUZMÁN, D.; HENRÍQUEZ, H.; GARCÍA, R.; ESCALONA, J. \& CIUFFARDI, I. Variante protrombótica C677T del gen metilenotetrahidrofolato reductasa es un biomarcador molecular de fallas de implantación en mujeres chilenas sometidas a protocolos de reproducción asistida. Int. J. Morphol., 28(1):65-69, 2010.

\section{MATERIAL Y METODO}

Población en estudio. Un total de 180 mujeres fueron incluidas en el estudio, 80 pacientes sometidas a procedimientos de fecundación asistida atendidas en el Instituto de Medicina Reproductiva de Concepción, Chile y 100 controles con historial de embarazos previos y ausencia de antecedentes de fallas reproductivas y trombosis. Datos demográficos e historia de hipertensión arterial, diabetes mellitus, dislipidemia y ciclos de reproducción asistida fallidos fueron obtenidos para cada paciente. Todas las participantes en el estudio firmaron un documento de consentimiento informado, de acuerdo a los principios básicos para investigación médica enumerados en la Declaración de Helsinki.

Genotipificación molecular. Se evaluaron las variantes G1691A del gen del factor V (F5, rs6025) G20210A del gen de la protrombina (F2, rs1799963) y C677T del gen de la enzima Metilenotetrahidrofolato reductasa (MTHFR, rs1801133), Para los análisis moleculares, el DNA genómico fue extraído de sangre periférica mediante técnica estandarizada (Salazar et al., 1998). La detección molecular de variantes en genes del factor $\mathrm{V}$ y factor II de la coagulación fue realizada por Reacción de la Polimerasa en Cadena seguida de restricción enzimática (PCR-RFLP) bajo condiciones descritas (Bertina et al., 1994; Poort et al., 1996). La genotipificación del polimorfismo MTHFR C677T se realizó utilizando partidores forward 5'CCGAAGCAGGGAGCTTTGAG-3' y reverse 5'AGGACGGTGCGGTGAGAGTG-3'. Las reacciones de amplificación fueron realizadas en un volumen final de 25 $\mu \mathrm{l}$ conteniendo $50 \mathrm{ng}$ de DNA genómico, $100 \mathrm{nM}$ de cada partidor, $200 \mathrm{mM}$ de cada dNTP, 1 unidad de Taq DNA polimerasa y buffer de PCR ( $\mathrm{KCl} 50 \mathrm{mM}, 2 \mathrm{mM} \mathrm{MgCl}, 20$ $\mathrm{mM}\left(\mathrm{NH}_{4}\right)_{2} \mathrm{SO}_{4}, 75 \mathrm{mM}$ Tris- $\left.\mathrm{HCl}, \mathrm{pH} 9.0\right)$.
Los productos de PCR fueron sometidos a restricción enzimática a $37^{\circ} \mathrm{C}$ por 12 horas con la endonucleasa respectiva. Los fragmentos de restricción fueron separados por electroforesis en gel de agarosa al $3 \%$ teñido con bromuro de Etidio y visualizado en transiluminador UV. Todos los geles fueron leídos por dos personas y el 20\% de los análisis fue repetido aleatoriamente a modo de control interno.

Análisis estadístico. Las frecuencias alélicas y genotípicas para cada variante fueron estimadas directamente por conteo de genes y las diferencias fueron evaluadas por test de chicuadrado. El Odds Ratio (OR) para falla de implantación en las pacientes sometidas a procedimientos de fecundación asistida que presentaron alguno de los polimorfismos analizados, fue calculado con un I.C. de $95 \%$, asumiendo significancia estadística con un valor de $\mathrm{p}<0.05$.

\section{RESULTADOS}

Las características clínicas y demográficas de pacientes con falla reproductiva y controles se presentan en la Tabla I. La distribución genotípica y frecuencia relativa de alelos para cada variante analizada se muestra en la Tabla II. Todas las frecuencias genotípicas se encontraban en equilibrio de Hardy-Weinberg.

Del total de mujeres incluidas en el estudio, sólo una paciente presentó el polimorfismo F5 G1691A al estado heterocigoto. Por su parte, se observó ausencia total de la variante F2 G20210A en la población estudiada. En el caso del polimorfismo MTHFR C677T, la frecuencia del alelo mutado fue significativamente diferente entre pacientes y controles $(p<0,03)$. La homocigocidad para la variante analizada en el gen MTHFR mostró asociación significativa con fallas de implantación (OR = 2,78; IC 1,147 - 6,755; p= 0,0199).

Tabla I. Características clínicas y demográficas de pacientes sometidas a protocolos de Fecundación asistida y controles.

\begin{tabular}{|c|c|c|c|}
\hline & Pacientes $n=80$ & Controles $n=100$ & \\
\hline Edad, años (Media + DS) & $31.9+4.17$ & $31.4+4.05$ & \\
\hline Diabetes Mellitus & $0(0 \%)$ & $0(0 \%)$ & \\
\hline Dislipidemia & $2(2.1 \%)$ & $0(0 \%)$ & \\
\hline Hipertensión & $0(0 \%)$ & $0(0 \%)$ & \\
\hline \multicolumn{4}{|l|}{ Procedimiento } \\
\hline FIV & $57(71.2 \%)$ & $0(0 \%)$ & \\
\hline ICSI & $19(23.8 \%)$ & $0(0 \%)$ & \\
\hline ICSI + Hatching asistido & $4(5.0 \%)$ & $0(0 \%)$ & \\
\hline Ciclos Fallidos & & & DS=Desviación Standard; \\
\hline 1 & $61(76.3 \%)$ & $0(0 \%)$ & $\begin{array}{l}\text { FIV=Fertilización in vitro; } \\
\text { ICSI=Invección Intracitoplasmát }\end{array}$ \\
\hline$\geq 2$ & $19(23.7 \%)$ & $0(0 \%)$ & Espermatozoides \\
\hline
\end{tabular}


GUZMÁN, N.; JARA, M. J.; MORALES, L.; SEPÚLVEDA, C.; GUZMÁN, D.; HENRÍQUEZ, H.; GARCÍA, R.; ESCALONA, J. \& CIUFFARDI, I. Variante protrombótica C677T del gen metilenotetrahidrofolato reductasa es un biomarcador molecular de fallas de implantación en mujeres chilenas sometidas a protocolos de reproducción asistida. Int. J. Morphol., 28(1):65-69, 2010.

Tabla II. Distribución genotípica y frecuencia relativa de alelos para las variantes F5 G1691A, F2 G20210A y MTHFR C677T en pacientes sometidas a protocolos de Fecundación Asistida y controles.

\begin{tabular}{lcccc}
\hline Polimorfismo & Genotipo & Pacientes $(\mathbf{n = 8 0})$ & Controles $(\mathbf{n = 1 0 0})$ & P* \\
\hline F5 & GG & $98.75 \%$ & $100 \%$ & NS \\
G1691A & GA & $1.25 \%$ & $0 \%$ & \\
(rs6025) & AA & $0 \%$ & $0 \%$ &
\end{tabular}

\begin{tabular}{lcccc} 
& Alelos & & & \\
& G & 0.975 & 1.00 & \\
F2 & A & 0.025 & 0.00 & \\
G20210A & GG & $100 \%$ & $100 \%$ & NS \\
(rs 1799963) & GA & $0 \%$ & $0 \%$ & \\
& AA & $0 \%$ & $0 \%$ & \\
& Alelos & & & $<0.02^{\mathrm{a}}$ \\
& G & 1.00 & 1.00 & \\
MTHFR & A & 0.00 & 0.00 & \\
C677T & CC & $30 \%$ & $30 \%$ & \\
$($ rs 1801133$)$ & CT & $50 \%$ & $8 \%$ & \\
& TT & $20 \%$ & 0.77 & \\
& Alelos & & 0.23 & \\
& C & 0.55 & & \\
\hline
\end{tabular}

${ }^{\mathrm{a}} \mathrm{OR}=2,78$ (IC $\left.1,147-6,755 ; p=0,0199\right) ; \mathrm{NS}=$ No significativo.

\section{DISCUSIÓN}

Las fallas de implantación constituyen uno de los principales inconvenientes en mujeres sometidas a procedimientos de fecundación asistida, no estando completamente dilucidados los mecanismos moleculares que median estas alteraciones. En este estudio, se evaluó la potencial asociación entre la presencia de tres variantes protrombóticas y fallas de implantación en mujeres chilenas sometidas a procedimientos de fecundación asistida y controles.

Los resultados muestran una baja frecuencia del polimorfismo F5 G1691A, además de ausencia total de la variante F2 G20210A, no encontrándose asociadas a fallas de implantación en la población estudiada. Estas frecuencias contrastan con las observadas en estudios realizados en población caucásica, en los que se ha descrito asociación con fallas de implantación (Grandone et al.; Azem et al.). En este sentido, diversos estudios muestran que estas variantes pre- sentarían elevadas frecuencias en población caucásica, estando prácticamente ausentes en poblaciones con un background genético distinto, como población negroide, asiática y amerindia (Herrmann et al., 1997; Zivelin et al., 1997; Garewal et al., 2003; Bauduer \& Lacombe, 2005). Así, la baja frecuencia de estos polimorfismos estaría relacionada con el background genético de la población chilena, la que presenta un componente predominantemente Amerindio (Rocco et al., 2002; García et al., 2006).

En contraste, destaca la elevada frecuencia de la variante MTHFR C677T, siendo significativamente mayor en el grupo de pacientes. Esto se correlaciona con estudios que muestran importantes variaciones en este polimorfismo, encontrándose frecuencias mayores en poblaciones latinoamericanas (Arruda et al., 1998; Camacho et al., 1998; Genoud et al., 2000). Interesantemente, la presencia del 
GUZMÁN, N.; JARA, M. J.; MORALES, L.; SEPÚLVEDA, C.; GUZMÁN, D.; HENRÍQUEZ, H.; GARCÍA, R.; ESCALONA, J. \& CIUFFARDI, I. Variante protrombótica C677T del gen metilenotetrahidrofolato reductasa es un biomarcador molecular de fallas de implantación en mujeres chilenas sometidas a protocolos de reproducción asistida. Int. J. Morphol., 28(1):65-69, 2010.

polimorfismo al estado homocigoto constituye un factor de riesgo de fallas de implantación en población chilena. El aumento del riesgo de fracaso de los protocolos de fecundación asistida en mujeres portadoras homocigotas del polimorfismo MTHFR C677T constituye el primer antecedente en Chile con respecto a esta asociación. Esto permite sugerir que la detección de esta variante en mujeres que serán sometidas a procedimientos de fecundación asistida constituiría una herramienta que podría mejorar el manejo clínico de las pacientes y tendría un impacto directo en la planificación y aplicación de los protocolos de reproducción asistida y ante el eventual pronóstico de embarazo. Estudios recientes han demostrado que la utilización de terapia con heparina de bajo peso molecular en dosis profilácticas en mujeres portadoras de polimorfismos protrombóticos, disminuiría el riesgo de fallas de implanta- ción y el desarrollo de complicaciones obstétricas, aumentando las tasas de embarazos exitosos (Huxtable et al., 2005; Pappalardo et al., 2005; Monastiri et al., 2007).

En resumen, los resultados muestran que la variante MTHFR C677T constituye un biomarcador molecular de riesgo de fallas de implantación en mujeres chilenas incluidas en protocolos de reproducción asistida. Así, su detección podría definir pacientes con riesgo elevado de falla reproductiva, permitiendo establecer estrategias de manejo clínico adecuadas para mejorar las tasas de éxito de este tipo de procedimientos.

AGRADECIMIENTOS: Este estudio fue financiado por un proyecto de la Dirección de Investigación (DIUSS $\mathrm{N}^{\circ}$ 04.1.9.8), Universidad San Sebastián, Chile.

GUZMÁN, N.; JARA, M. J.; MORALES, L.; SEPÚLVEDA, C.; GUZMÁN, D.; HENRÍQUEZ, H.; GARCÍA, R.; ESCALONA, J. \& CIUFFARDI, I. Prothrombotic variant C677T at the methylenetetrahydrofolate reductase gene is a molecular biomarker of implantation failure in Chilean women undergoing assisted reproductive protocols. Int. J. Morphol., 28(1):65-69, 2010.

SUMMARY: Implantation failures are considered an important cause of infertility in women undergoing assisted reproductive protocols. Recent evidence suggests that the presence of prothrombotic variants is associated with obstetric defects and reproductive failure. However, results between several populations are contradictory. According this evidence, we evaluated the potential association between prothrombotic variants and implantation failure susceptibility in Chilean women undergoing Assisted Reproductive protocols. 180 women, 80 patients undergoing assisted reproductive protocols and 100 controls were included in this study. Molecular genotyping of prothrombotic variants in candidate genes was realized by PCR-RFLP. We observed one patient heterozygote for factor V G1691A variant, and total absence to prothrombin G20210A polymorphism in patients and controls. Genotype distribution and allelic frequencies of MTHFR C677T polymorphism were significantly different between patients and controls $(\mathrm{p}<0.05)$. Odss Ratio for implantation failure associated to homozygote genotype was 2.78 (95\% IC $1.147-6.755 ; p=0.0199$ ). In summary, our data suggest that the MTHFR C677T polymorphism constituted a molecular biomarker of implantation failure susceptibility in Chilean population.

KEY WORDS: Infertility; Implantation failure; Biomarker.

\section{REFERENCIAS BIBLIOGRÁFICAS}

Arruda, V.; Siqueira, L.; Gonçalves, M.; von Zuben, P.; Soares, M.; Menezes, R.; Annichino-Bizzacchi, J. \& Costa, F. Prevalence of the mutation C677>T in the methylene tetrahydrofolate reductase gene among distinct ethnic groups in Brazil. Am. J. Med. Genet., 78: 332-5, 1998.

Azem, F.; Many, A.; Ben Ami, I.; Yovel, I.; Amit, A.; Lessing, J. \& Kupferminc, M. Increased rates of thrombophilia in women with repeated IVF failures. Hum. Reprod., 19:368-70, 2004.

Bauduer, F. \& Lacombe, D. Factor V Leiden, prothrombin 20210A, methylenetetrahydrofolate reductase $677 \mathrm{~T}$ and population genetics. Mol. Genet. Metab., 86:91-9, 2005.
Bertina, R. M.; Koeleman, B. P. C.; Koster, T.; Rosendaal, F. R.; Dirven, R. J.; de Ronde, H.; van der Velden, P. A. \& Reitsma, P. H. Mutation in blood coagulation factor $\mathrm{V}$ associated with resistance to activated protein $\mathrm{C}$. Nature, 369:64-7, 1994.

Buchholz, T. \& Thaler, C. Inherited thrombophilia: impact on human reproduction. Am. J. Reprod. Immunol., 50:2032, 2003.

Camacho, O.; Giusti, B.; Restrepo Fernandez, C. M.; Abbate, R. \& Pepe, G. Frequency of factor V (FV) Leiden and C677T Methylenetetrahydrofolate reductase (MTHFR) mutation in Colombians. Thromb. Haemost., 79:883-4, 1998. 
GUZMÁN, N.; JARA, M. J.; MORALES, L.; SEPÚLVEDA, C.; GUZMÁN, D.; HENRÍQUEZ, H.; GARCÍA, R.; ESCALONA, J. \& CIUFFARDI, I. Variante protrombótica C677T del gen metilenotetrahidrofolato reductasa es un biomarcador molecular de fallas de implantación en mujeres chilenas sometidas a protocolos de reproducción asistida. Int. J. Morphol., 28(1):65-69, 2010.

García, F.; Moraga, M.; Vera, S.; Henríquez, H.; Llop, E.; Aspillaga, E. \& Rothhammer, F. mtDNA microevolution in Southern Chile's archipelagos. Am. J. Phys. Anthropol., 129:473-81, 2006.

Garewal, G.; Das, R.; Varma, S.; Chawla, Y. \& Prabhakar, $\mathrm{S}$. Heterogeneus distribution of Factor $\mathrm{V}$ Leiden in patients from north India with venous thromboembolism. J. Throm.b Haemost., 1:1329-30, 2003.

Genoud, V.; Castañon, M.; Annichino-Bizzacchi, J.; Korin, J. \& Kordich, L. Prevalence of three prothrombotic polymorphisms: factor V G1691A, factor II G20210A and methylenetetrahydrofolate reductase (MTHFR) C677T in Argentina. Thromb. Res., 100:127-31, 2000.

Grandone, E.; Colaizzo, D.; Lo Blue, A.; Checola, M.; Cittadini, E. \& Margaglione, M. Inherited thrombophilia and in vitro fertilization implantation failure. Fertil. Steril., 76:201-2, 2001.

Herrmann, F.; Koesling, M.; Schröder, W.; Altman, R.; Jiménez Bonilla, R.; Lopaciuk, S.; Perez-Requejo, J. L. $\&$ Singh, J. R. Prevalence of factor V Leiden mutation in various populations. Genet. Epidemiol., 14:403-11, 1997.

Huxtable, L.; Tafreshi, M. \& Ondreyco, S. A Protocol for the use of Enoxaparin during pregnancy: Results from 85 pregnancies including 13 multiple gestation pregnancies. Clin. Appl. Thromb. Hemost., 11:171-81, 2005 .

Macklon, N.; Geraedts, J. \& Fauser, B. Conception to ongoing pregnancy: the "black box" of early pregnancy. Hum. Reprod. Update., 4:333-43, 2002.

McIntyre, J. Antiphospholipid antibodies in implantation failures. Am. J. Reprod. Immunol., 49:221-9, 2003.

Monastiri, K.; Seket, B.; Chouchane, S.; Samama, M. M. \& Mahjoub, T. Are low doses of enoxaparin with aspirin sufficient to guarantee good gestational outcome in women with heterozygote factor V Leiden mutation? Eur. J. Obstet. Gynecol. Reprod. Biol., 130:138-40, 2007.

Pappalardo, C.; Sarto, A.; Donaldson, M.; Paganini, A. \& Pasqualini, R. S. Enoxaparin in oocyte donation cycles in women with thrombophilia: improvement in pregnancy and implantation rates. Throm.b Res., 115S: 139, 2005.

Poort, S. R.; Rosendaal, F.; Reitsma, P. \& Bertina, R. A common genetic variation in the 3 -untranslated region of the prothrombin gene is associated with elevated plasma prothrombin levels and increase in venous thrombosis. Blood, 88:3698-703, 1996.

Red-Horse, K.; Zhou, Y.; Genbacev, O.; Prakobphol, A.; Foulk, R.; McMaster, M. \& Fisher, S. Trophoblast differentiation during embryo implantation and formation of the maternal-fetal interface. J. Clin. Invest., 114:744-54, 2004.

Rocco, P.; Morales, C.; Moraga, M.; Miquel, J.; Nervi, F.; Llop, E.; Carvallo, P. \& Rothhamer, F. Composición genética de la población chilena. Distribución de polimorfismos de DNA mitocondrial en grupos originarios y en la población mixta de Santiago. Rev. Méd. Chile., 130:125-31, 2002.

Salazar, L. A.; Hirata, M.; Cavalli, S.; Machado, M. O. \& Hirata, R. D. Optimized procedure DNA isolation from fresh and cryopreserved clotted human blood useful in clinical molecular testing. Clin. Chem., 44:1748-50, 1998.

Villarreal, C.; García-Aguirre, G.; Hernández, C.; Vega, O.; Borbolla, J. R. \& Collados, M. Congenital Thrombophilia associated to obstetric complications. $J$. Thromb. Thrombolysis, 14:163-9, 2002.

Wolf, C.; Haubelt, H.; Ulrich, H.; Hinney, B.; Krome-Cesar, C.; Legler, T.; Hellstern, P.; Emons, G.; Zoll, B. \& Köhler, M. Recurrent pregnancy loss and its relation to $\mathrm{FV}$ Leiden, FII G20210A and polymorphisms of plasminogen activator and plasminogen activator inhibitor. Pathophysiol. Haemos. Thromb., 33:134-7, 2003.

Zinaman, M. J.; Clegg, E. D.; Brown, C. C.; O'Connor, J. \& Selevan, S. G. Estimates of human fertility and pregnancy loss. Fertil. Steril., 65:503-9, 1996.

Zivelin, A.; Griffin, J.; Xu, X.; Pabinger, I.; Samama, M.; Conard, J.; Brenner, B.; Eldor, A. \& Seligsohn, U. A single genetic origin for a common Caucasian risk factor for venous thrombosis. Blood, 89:397-402, 1997.

Dirección para correspondencia:

Neftalí Guzmán

Laboratorio de Diagnóstico Molecular,

Facultad de Ciencias de la Salud,

Universidad San Sebastián

CHILE

Email:nguzman@uss.cl

Recibido : 30-10-2009 Aceptado: 22-12-2009 
